\title{
Correção cirúrgica da coartação da aorta nos primeiros seis meses de vida
}

\author{
Luciano Cabral ALBUQUERQUE*, Marco Antônio GOLDANI*, Juremir João GOLDANI*, Rubens \\ Lorentz ARAÚJO*, Ricardo Medeiros PIANTÁ* ${ }^{*}$ Luciane Barreneche NARVAES*, Júlia de Barros \\ MACHADO*, Jeferson AITA*, João Batista PETRACCO*
}

RBCCV 44205-583

Albuquerque L C, Goldani M A, Goldani J J, Araújo R L, Piantá R M, Narvaes L B, Machado J B, Aita J, Petracco J B - Correção cirúrgica da coartação da aorta nos primeiros seis meses de vida. Rev Bras Cir Cardiovasc 2002; 17(2): 29-35.

RESUMO: Objetivo: Avaliar os resultados imediatos e tardios da correção cirúrgica da coartação da aorta torácica (CoAo) nos primeiros seis meses de vida.

Casuística e Métodos: Entre janeiro de 1994 e maio de 2001, 89 pacientes foram submetidos à correção de CoAo pelas técnicas de aortoplastia com flap de subclávia (Grupo $1 / n=49$ ), e resseção com anastomose término-terminal (Grupo $2 / n=40$ ). Foram analisadas e comparadas as seguintes variáveis: idade no momento da operação, presença de anomalias intracardíacas, gradiente de pressão pré e pós-operatório, complicações e mortalidade cirúrgicas, bem como sobrevida tardia livre de eventos.

Resultados: Houve predomínio do sexo masculino $(n=60-68 \%)$ e a grande maioria dos casos foram operados no primeiro mês de vida, por insuficiência cardíaca (IC) refratária ( $n=62$ $70 \%$ ). A CoAo apresentou-se isolada em $23(26 \%)$ pacientes, e associada a defeitos intracardíacos em $66(74 \%)$. O gradiente médio pré e pós-operatório foi, respectivamente, de $42 \mathrm{mmHg}$ e 4,5 $\mathrm{mmHg}$, não havendo diferença entre os grupos. As complicações cirúrgicas mais freqüentes foram hipertensão arterial persistente $(n=27)$, estenose residual $(n=5)$ e sangramento $(n=3)$, e a mortalidade operatória foi de $10,2 \%(n=9)$, sendo significativamente maior nos casos com cardiopatias associadas ( $12 \%$ vs. $4 \%$ - $p<0,05)$. Não houve diferença na incidência de complicações e mortalidade entre os grupos. A ocorrência de recoartação tardia foi de $16 \%$ no grupo 1 e $15 \%$ no grupo 2 ( $p=N S$ ), e a taxa de sobrevida livre de eventos em 60 meses foi de $76 \%$ e $81 \%$, respectivamente $(p=N S)$.

Conclusões: A maioria dos casos de CoAo manifesta nos primeiros meses de vida requer correção ainda precocemente, por IC refratária. A mortalidade cirúrgica é significativa naqueles pacientes com defeitos intracardíacos; não houve diferenças na morbi-mortalidade e na incidência de recoartação, entre as técnicas cirúrgicas empregadas.

DESCRITORES: Coartação aórtica, cirurgia. Aorta torácica, anormalidades

Trabalho realizado no Serviço de Cirurgia Cardiovascular do Hospital São Lucas - Faculdade de Medicina da PUCRS. Porto Alegre, RS, Brasil.

Recebido para publicação em dezembro de 2001.

* Do Hospital São Lucas - Faculdade de Medicina da PUCRS.

Endereço para correspondência: Luciano Cabral Albuquerque. Centro Clínico do HSL-PUCRS. Av. Ipiranga 6690, conj. 615. Porto Alegre, RS, Brasil. CEP 90610-000. Tel.: (51)3336-8190 - Fax: (51)3339-9040. e-mail: alb.23@ terra.com.br 


\section{INTRODUÇÃO}

A Coartação da aorta torácica (CoAo), quando clinicamente sintomática nos primeiros meses de vida, em geral, manifesta-se por insuficiência cardíaca grave e por vezes refratária, necessitando tratamento cirúrgico precoce ${ }^{(1,2)}$.

Historicamente, a correção nessa faixa etária tem revelado consideráveis taxas de mortalidade, estenose residual, e recoartação tardia, além de envolver dificuldades técnicas peculiares ${ }^{(3-5)}$.

Em 1945, CRAFOORD \& NYLIN (6) descreveram o reparo cirúrgico da CoAo na infância, através da ressecção seguida de anastomose término-terminal (T-T), técnica considerada de fácil reprodutibilidade nas crianças maiores e nos adultos, mas acompanhada de alto grau de estenose residual no recémnascido e no lactente $(7,8)$.

Em 1966, WALDHAUSEN \& NAHRWOLD (5) introduziram a aortoplastia com flap de subclávia esquerda como técnica preferencial de correção na infância, propondo vantagens como menor extensão de dissecção, preservando circulação colateral, e um menor potencial de re-estenose circunferencial.

Em nosso meio, relevante contribuição foram os estudos de MEIER et al. ${ }^{(9)}$, que introduziram a aortoplastia com mobilização extensa da artéria subclávia esquerda, sem necessidade de sua ligadura ou sacrifício de artérias intercostais, técnica bastante aplicável a crianças maiores e adultos jovens.

Além disso, alguns autores nos anos 70 apresentaram sua experiência utilizando patch de PTFE ou Dacron (10,11) para ampliação da zona de estreitamento, estratégia posteriormente abandonada em crianças menores, pelo desenvolvimento tardio de pseudo-aneurismas ${ }^{(12,13)}$. Mais recentemente, a constatação de que a remoção do tecido embriologicamente relativo ao ductus arteriosus diminui as chances de recoartação (14-16) tem levado alguns grupos a proporem uma ressecção mais ampliada da aorta acometida, e anastomose T-T com fio absorvível (17-20).

O objetivo do presente estudo é apresentar a experiência de 7 anos do Serviço de Cirurgia Cardiovascular do Hospital São Lucas da PUCRS, no tratamento cirúrgico da CoAo nos primeiros 6 meses de vida, utilizando as técnicas de aortoplastia com flap de subclávia ou ressecção com anastomose término-terminal.

\section{CASUÍSTICA E MÉTODOS}

Foram analisados, retrospectivamente, os prontuários de 89 pacientes menores de 6 meses de idade, $60(68 \%)$ do sexo masculino e $29(32 \%)$ do feminino, submetidos à correção de CoAo entre janeiro de 1994 e maio de 2001.

No grupo 1, 49 pacientes foram submetidos à técnica de aortoplastia com flap de subclávia esquerda, através de toracotomia no $4^{\circ}$ espaço intercostal esquerdo (EICE). Foram amplamente dissecadas a artéria subclávia, a aorta descendente proximal e a porção distal do arco transverso, e o ductus arteriosus foi ligado, quando presente. Após a infusão de heparina $1000 \mathrm{U} / \mathrm{kg}$, a artéria subclávia foi seccionada distalmente e incisada no sentido longitudinal, transpondo a zona de estreitamento. Foram procedidas a ressecção de parte do tecido endotelial responsável pela coartação, e a rotação da subclávia, empregando-se sutura contínua de polipropileno (Prolene®) ou poligliconato (PDS $®$ ) para a istmoplastia.

O grupo 2 foi constituído de 40 pacientes, nos quais a técnica de correção realizada foi a ressecção completa da zona de CoAo, seguida de anastomose T-T. Mediante toracotomia no $4^{\circ}$ EICE, procedeu-se à dissecção ampliada do arco transverso e da aorta descendente proximal, com ligadura de 2 a 4 artérias intercostais e mobilização maior da artéria subclávia. $\mathrm{O}$ ductus arteriosus foi ligado, quando indicado, e infundida heparina na dose de $1000 \mathrm{U} / \mathrm{kg}$. Após ressecção transversal da zona de coartação, foi realizada sutura contínua com fio de polipropileno (Prolene $\AA$ ) ou de poligliconato (PDS $\AA$ ).

Em ambos os grupos, até o ano de 1995, utilizamos fio de polipropileno 6 ou 7-0 (16 casos), quando passamos a ter disponível o fio absorvível de poligliconato 6 ou 7-0, utilizado como rotina a partir de então (73 casos).

Nos casos de comunicação interventricular associada (CIV), realizamos a bandagem da artéria pulmonar no mesmo ato operatório, após o desclampeamento da aorta.

O Gráfico 1 apresenta a freqüência anual das duas técnicas empregadas, no qual podemos observar que no início dessa experiência houve um claro predomínio da técnica de Waldhausen, ao passo que atualmente essa distribuição já é equivalente.

Cardiopatias intracardíacas associadas ocorreram em 66 casos, sendo as mais freqüentes CIV $(n=34)$, comunicação interatrial $(n=27)$, hipoplasia do arco aórtico $(n=7)$, valva aórtica bicúspide e/ou estenose aórtica $(n=5)$ e transposição das grandes artérias $(n=2)$.

\section{GRÁFICO 1 \\ DISTRIBUIÇÃO DAS TÉCNICAS EMPREGADAS DURANTE O PERÍODO DO ESTUDO.}

\section{Distribuição anual}

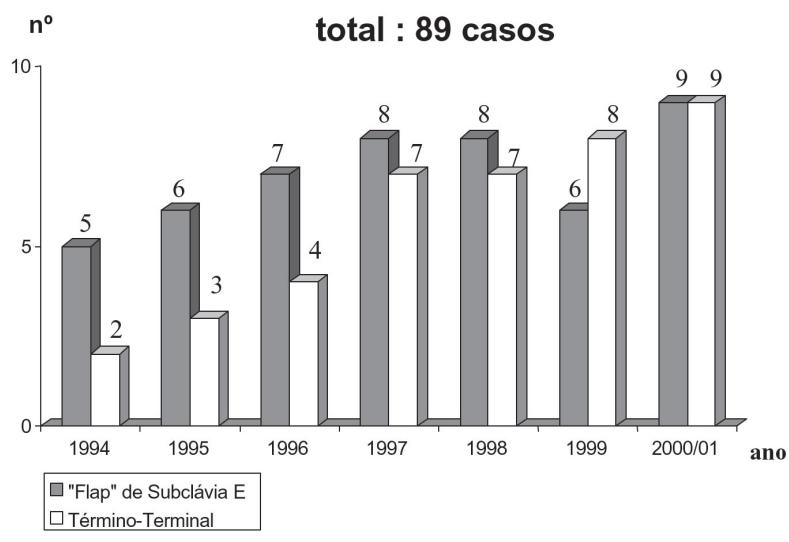


O acompanhamento pós-alta hospitalar constou de revisões semestrais com realização de ecocardiograma bidimensional para controle do gradiente, e internações intercorrentes, quando necessárias.

Foram ainda analisadas e comparadas, entre os grupos, as variáveis gradiente de pressão residual, taxas de mortalidade cirúrgica e recoartação tardia, e sobrevida livre de eventos.

Para análise estatística, os dados foram submetidos ao teste $\mathrm{T}$ de Student para amostras independentes, e ao teste exato de Fischer, considerando-se significativo 0 valor de $\mathrm{p}<0,05$.

\section{RESULTADOS}

Todos os pacientes encontravam-se internados em Unidade de Tratamento Intensivo (UTI) Neonatal ou Pediátrica, gravemente sintomáticos, e foram diagnosticados pelo ecocardiograma bidimensional, o qual revelou um gradiente médio de pressão através da coartação de $41,7 \mathrm{mmHg}$ (31 a $62 \mathrm{mmHg}$ ). A idade no momento da operação variou de 1 a 176 dias (mediana 27 dias), sendo $70 \%$ dos casos operados ainda no período neonatal, por insuficiência cardíaca congestiva refratária (Gráfico 2).

Em $75 \%$ da amostra $(n=66)$, havia associação de outros defeitos intracardíacos, enquanto apenas $25 \%$ dos pacientes $(n=23)$ apresentavam CoAo isoladamente

\section{GRÁFICO 2 \\ DISTRIBUIÇÃO ETÁRIA NO MOMENTO DA INDICAÇÃO DA OPERAÇÃO}

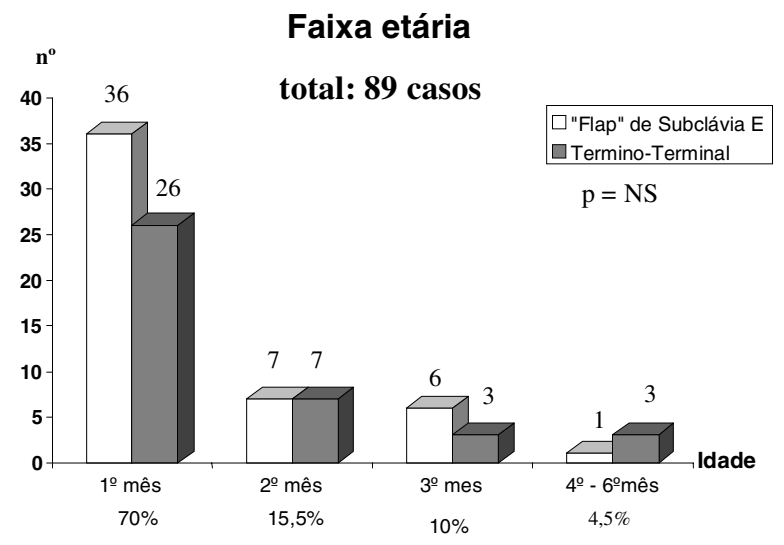

$(p<0,05)$. O tempo médio de permanência em UTI foi de 3,7 dias (1 a 34 dias), e o tempo médio de internação 12,6 dias (6 a 34 dias), não havendo diferença significativa entre os grupos.

O gradiente residual médio de pressão, no pós-operatório intra-hospitalar, foi de $6 \mathrm{mmHg}$ no grupo 1 e de 3,9 $\mathrm{mmHg}$ no grupo 2 ( $\mathrm{p}=\mathrm{NS}$ ), tendo ocorrido 5 casos de estenose residual (definida por gradiente ecocardiográfico igual ou superior a $20 \mathrm{mmHg}$ ), 3 no grupo 1 e 2 no grupo
2 ( $p=N S)$. As demais complicações pós-operatórias relativas ao procedimento foram hipertensão arterial persistente $(30 \%)$ e sangramento com necessidade de reintervenção $(3,4 \%)$. Não houve ocorrência de isquemia grave nem de atrofia do membro superior esquerdo nos casos submetidos à técnica de Waldhausen, nem de paraplegia na técnica de ressecção com anastomose T$\mathrm{T}$.

A mortalidade cirúrgica global foi de 10,1\% ( $n=9)$, sendo relacionada em 8 pacientes a complicações de cardiopatias estruturais associadas, e à infecção respiratória em recém-nascido de baixo peso $(1980 \mathrm{~g})$ em 1 caso com CoAo isolada, não havendo diferença significativa entre os grupos. A razão de chances para óbito intrahospitalar em pacientes com cardiopatias associadas foi de 2,8 (Gráfico 3).

No seguimento tardio médio foi de 42 meses ( 3 a 60 meses), a taxa de recoartação (definida como gradiente ecocardiográfico igual ou superior a $20 \mathrm{mmHg}$ ) foi de $15,7 \%$, sendo 8 casos no grupo 1 e 6 casos no grupo 2 $(\mathrm{p}=\mathrm{NS}$ ). A probabilidade de sobrevivência livre de eventos em 60 meses foi de $76 \%$ no grupo 1 e $81 \%$ no grupo 2 ( $\mathrm{p}=\mathrm{NS}-$ Gráfico 4).

\section{GRÁFICO 3 \\ MORTALIDADE CIRÚRGICA DA COAO EM CASOS COM E SEM DEFEITOS INTRACARDÍACOS ASSOCIADOS}
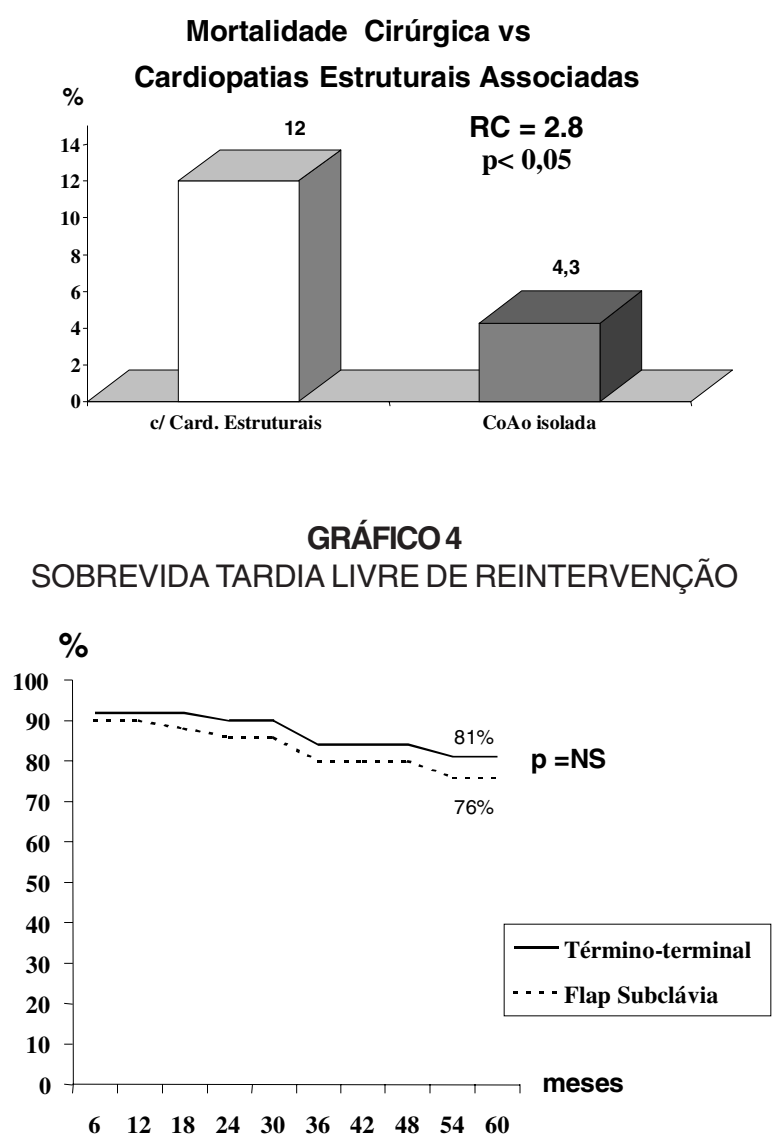
Dos 14 casos de recoartação, 8 foram no grupo 1 e 6 no grupo 2 ( $p=N S$ ); 9 foram submetidos à angioplastia percutânea por cateter-balão, com sucesso, 3 foram reoperados (todos com interposição de enxerto de PTFE) e 2 morreram antes de intervenção.

\section{COMENTÁRIOS}

Quando clinicamente evidente nos primeiros meses de vida, a CoAo em geral se apresenta com IC refratária, freqüentemente acompanhada de falência renal e acidose metabólica. A alta mortalidade relacionada a esse quadro de hipoperfusão distal e hipertensão arterial sustentadas exige imediata intervenção para alívio do processo obstrutivo.

Não obstante o consenso sobre a necessidade de correção no momento do diagnóstico, a técnica de escolha para o reparo da CoAo sintomática nessa fase da vida permanece controversa, principalmente em relação à incidência de recoartação tardia, que na literatura é tão variável como entre 4 a $60 \%{ }^{(21-24)}$. Em crianças abaixo de 2 anos, as técnicas mais freqüentemente utilizadas são a aortoplastia com flap de subclávia (WALDHAUSEN \& NAHRWOLD ${ }^{(5)}$ ) e a ressecção com anastomose T$T$ (CRAFOORD \& NYLIN ${ }^{(6)}$ ).

A favor da técnica de WALDHAUSEN \& NAHRWOLD ${ }^{(5)}$, diversos autores advogam sua mais rápida e fácil execução em crianças menores, o não sacrifício de intercostais e o menor potencial de reestenose circunferencial. Entretanto, sua maior limitação tem sido as repercussões circulatórias no membro superior esquerdo (MSE), que podem levar à perda ou atrofia da extremidade, descrita em algumas séries ${ }^{(25-26)}$. Felizmente, esta complicação tem ocorrido em menos de $1 \%$ dos casos, e, segundo autores como FENCHEL et al. (27) e HAN et al. (28), pode ser minimizada com a ligadura rotineira da origem da artéria vertebral, manobra que também evita o desenvolvimento de síndrome do roubo de subclávia.

No trabalho de HAN et al. (28), em 126 neonatos submetidos à aortoplastia com flap de subclávia, não houve nenhum caso de isquemia grave de MSE; as complicações inerentes ao procedimento foram quilotórax $(n=4)$, lesão do nervo laringeo recorrente $(n=1)$, paralisia frênica $(n=1)$ e síndrome de Horner $(n=1)$. A mortalidade hospitalar foi de $11 \%$ (nenhum óbito em CoAo isolada) e $10 \%$ dos pacientes apresentaram recorrência da coartação no seguimento tardio. Alguns detalhes técnicos enfatizados, que sustentam estes bons resultados, são a remoção completa do "degrau" formado pelo tecido de proliferação miointimal ao nível da estenose, e a ampliação da aortotomia longitudinal até bem abaixo do sítio de estreitamento e do ductus remanescente.

Em defesa da técnica de ressecção e anastomose $\mathrm{T}$ - $\mathrm{T}$, os argumentos mais consistentes são o não sacrifício da artéria subclávia e a remoção completa do tecido ductal, histologicamente relacionado à gênese da recoartação e considerado por alguns autores como o maior determinante de recorrência.

CONTE et al. (29) utilizaram a ressecção ampliada seguida de anastomose T-T em 307 pacientes no período neonatal, a maioria dos quais com outros defeitos intracardíacos $(70 \%)$. A mortalidade cirúrgica global foi de $17 \%$, mas de apenas $2 \%$ nos casos de CoAo isolada; a baixa incidência para a faixa etária de recoartação tardia (10\%) foi resolvida com sucesso por angioplastia transluminal percutânea (ATP), e a probabilidade de sobrevida livre de reoperação em 10 anos foi de $93 \%$, reforçando a indicação pelos autores desta abordagem.

Mais recentemente, VAN SON et al. ${ }^{(16)}$ analisaram o tecido aórtico removido de 25 pacientes com idade média de 22 dias, submetidos a ressecção ampliada da CoAo, sem gradiente de pressão residual no ecocardiograma ou HAS no pós-operatório (supostamente "bem corrigidos"). Todas as amostras histológicas demonstraram a presença de tecido ductal na aorta descendente, com extensã̉o entre 3,9 a 5,2 $\mathrm{mm}$, que se projetava em contiguidade à parede lateral ou à região do istmo, corroborando os achados descritos por outros autores $(8,14,15,17,19,20,29,30)$.

Em nossa experiência, antes de 1994, a técnica de WALDHAUSEN \& NAHRWOLD ${ }^{(5)}$ era utilizada quase que exclusivamente em crianças menores com CoAo sintomática, havendo um considerável "conforto" dos cirurgiões com essa abordagem. Como demonstrado no Gráfico 1, iniciamos, timidamente nessa época, a realizar a ressecção e anastomose T-T, ao passo que nos últimos 4 anos, a afinidade por uma ou outra técnica foi substituída por critérios anatômicos individualizados, na tomada de decisão intra-operatória. Atualmente, através da análise de fatores como idade e peso da criança, extensão da zona de coartação, padrão de distribuição das artérias intercostais, diâmetro da subclávia e da aorta descendente, grau de estabilidade hemodinâmica, e necessidade de procedimentos associados, optamos por qual das técnicas descritas deve ser empregada, desde que, se realizada a operação de WALDHAUSEN \& NAHRWOLD ${ }^{(5)}$, proceda-se a remoção do "degrau" de tecido de proliferação miointimal.

Nossos resultados não apontam diferenças significativas entre as técnicas nos desfechos estudados: a mortalidade intra-hospitalar foi $10,2 \%$ vs. $10 \%$, a ocorrência de recoartação tardia foi $16,6 \%$ vs. $15 \%$, e a probabilidade de sobrevida livre de reintervenção em 60 meses foi de $76 \%$ vs. $81 \%$, respectivamente para os grupos 1 e 2 .

Achados semelhantes foram reportados por COBANOGLU et al. ${ }^{(31,32)}$, que em sua série mais recente, estudaram 86 pacientes abaixo de 3 meses submetidos à aortoplastia com flap de subclávia $(n=47)$ ou à ressecção e anastomose T-T $(n=39)$. Houve comparável taxa de mortalidade precoce $(8,5 \%$ vs. $5,1 \%)$, recoartação (10,5\% para ambas) e sobrevida livre de 
reoperação em 10 anos (90\% vs. 86\%). Os autores ressaltam que a recoartação, embora com incidência semelhante, ocorre por diferentes mecanismos: por tensão excessiva na linha de sutura na anastomose TT (mais do que pela utilização ou não de fio absorvível, e pela sutura contínua ou com pontos separados), e pela proliferação de tecido ductal não ressecado, na técnica de WALDHAUSEN \& NAHRWOLD ${ }^{(5)}$.

Na verdade, devemos entender a coartação da aorta na infância como uma entidade anátomo-patológica complexa, que requer o mesmo cuidado dispensado às cardiopatias consideradas como mais graves, tanto no aspecto de planejamento cirúrgico, quanto de cuidados intensivos pós-operatórios. Ainda hoje, não obstante o aprimoramento técnico e cirúrgico, a mortalidade da correção cirúrgica precoce permanece entre $5 \%$ e $25 \%$ na maioria dos grupos, incrementada em até 10 vezes pela associação com outros defeitos intracardíacos, em especial a CIV (28-30,32,33).

As complicações cirúrgicas ainda relevantes permanecem sendo a hipertensão arterial sistêmicas (HAS) persistente e a recoartação a médio e longo prazo. A incidência de HAS tem sido descrita como tão alta quanto $60 \%$, mesmo em casos sem gradiente residual ${ }^{(3)}$, constituindo a mais comum intercorrência clínica nesses pacientes, e tendo como principal preditor de risco o tempo prolongado de exposição da porção cranial do organismo à pressão elevada, o que levaria à acomodação do reflexo barorreceptor e à diminuição da complacência da aorta proximal, muitas vezes irreversíveis; nesse sentido, o uso agressivo de prostaglandina E1 para tentar otimizar o estado hemodinâmico pré-operatório é recomendado ${ }^{(16)}$.

Em relação à recoartação tardia, sua ocorrência em $15,7 \%$ de nossos pacientes é condizente com a apontada nos trabalhos mais recentes ${ }^{(16,28-30,32,33)}$, nossa política em seu manejo tem sido indicar a angioplastia percutânea, e na série descrita a abordagem foi satisfatória em promover o alívio de sintomas e redução do gradiente para menos de $20 \mathrm{mmHg}$ em todos os casos em que a técnica foi utilizada $(n=9)$. Embora tenha sido claramente demonstrado que a ATP de CoAo nativa apresenta uma taxa superior a $40 \%$ de re-estenose significativa em 2 anos (P1,P2,P3), os resultados da técnica têm sido bastante promissores no manejo da recorrência pós-cirúrgica, principalmente por envolver menores riscos.

\section{CONCLUSÃO}

Em conclusão, podemos afirmar que a coartação da aorta quando clinicamente manifesta nos primeiros meses de vida, em geral determina insuficiência cardíaca refratária e óbito, se não corrigida precocemente. Em nossa amostra, as técnicas de aortoplastia com flap de subclávia e ressecção com anastomose T-T apresentaram resultados comparáveis em termos de eficácia, mortalidade intra-hospitalar e incidência de recoartação a longo prazo. A mortalidade cirúrgica da correção da CoAo nessa faixa etária permanece expressiva, e está diretamente relacionada à presença de defeitos intracardíacos associados.

RBCCV 44205-583

Albuquerque L C, Goldani M A, Goldani J J, Araújo R L, Piantá R M, Narvaes L B, Machado $\mathrm{J}$ B, Aita J, Petracco J B - Surgical repair of aortic coarctation under than six months of age. Rev Bras Cir Cardiovasc 2002; 17(2): 28-34.

ABSTRACT: Objective: To evaluate early and late results of aortic coarctation (CoAo) surgical repair in early months of life.

Material and Methods: Between January 1994 and May 2001, 89 patients were submitted to CoAo repair by subclavian artery flap angioplasty (Group $1-n=49$ ) or resection with end-to-end anastomosis (Group $2-n=40$ ). We analyzed and compared the mean age at operation, associated cardiac malformations, pre and postoperative aortic pressure gradient, surgical complications and mortality, and actuarial freedom from events curve.

Results: Most of patients were male $(n=60-68 \%)$ and underwent to aortic repair under than one month of age, with severe heart failure $(n=62-70 \%)$. CoAo was associated with intracardiac defects in 66 cases (74\%). The aortic pressure gradient before and after surgery was $42 \mathrm{mmHg}$ and $4,5 \mathrm{mmHg}$, respectively. Postoperative complications were persistent arterial hypertension $(n=27)$, residual stenosis $(n=5)$ and bleeding $(n=3)$, and operative mortality was $10,2 \%(n=9)$, markedly higher in patients with associated cardiac malformations $(12 \% v s .4 \%-p<0,05)$. There were no differences in complications and mortality in both groups. Late re-coarctation was $16 \%$ in group 1 and $15 \%$ in group 2 (NS) and actuarial freedom from events in 60 months was $76 \%$ and $81 \%$, respectively (NS).

Conclusions: Most of surgical repair in symptomatic CoAo during early months is a lifesaving procedure, due to severe heart failure; operative mortality is significant in patients with others cardiac defects; there were no differences in morbidity, mortality and recurrent coarctation rate between two surgical techniques performed.

DESCRIPTORS: Aortic coarctation, surgery. Aorta, thoracic, abnormalities. 


\section{REFERÊNCIAS BIBLIOGRÁFICAS}

1. Shinebourne EA, Tam AS, Elseed AM Paneth M, Lennox SC, Cleland WP - Coarctation of the aorta in infancy and childhood. Br Heart J 1976; 38: 375-80.

2. Yee ES, Soifer SJ, Turley K, Verrier ED, Fishman NH, Ebert PA - Infant coarctation: a spectrum in clinical presentation and treatment. Ann Thorac Surg 1986; 42: 488-93.

3. Cohen M, Fuster V, Steele PM, Driscoll D, McGoon DC - Coarctation of the aorta: long-term followup and prediction of outcome after surgical correction. Circulation 1989; 80: 840-5.

4. Quaegebeur JM, Jonas RA, Weinberg AD, Blackstone EH, Kirklin JW - Outcomes in seriously ill neonates with coarctation of the aorta: a multiinstitutional study. J Thorac Cardiovasc Surg 1994; 108: 841-54.

5. Waldhausen JA \& Nahrwold DL - Repair of coarctation of the aorta with a subclavian flap. $J$ Thorac Cardiovasc Surg 1966; 51: 532-3.

6. Crafoord C \& Nylin G - Congenital coarctation of the aorta and its surgical treatment. $J$ Thorac Cardiovasc Surg 1945; 14: 347-61.

7. Körfer R, Meyer H, Kleikamp G, Bircks W Early and late results after resection and end-to-end anastomosis of coarctation of the thoracic aorta in early infancy. J Thorac Cardiovasc Surg 1985; 89: 61622.

8. Van Son JA, Daniels O, Vicent JG, Van Lier HJ, Lacquet LK - Appraisal of resection and end-toend anastomosis for repair of coarctation of the aorta in infancy: preference for resection. Ann Thorac Surg 1989; 48: 496-502.

9. Meier MA, Lucchese FA, Jazbik W, Nesralla IA, Mendonça JT - A new technique for repair of aortic coarctation: subclavian flap aortoplasty with preservation of arterial blood flow to the left arm. $J$ Thorac Cardiovasc Surg 1986; 92:1005-12.

10.Reul GJ Jr, Kabbani SS, Sandiford FM, Wukasch DC, Cooley DA - Repair of coarctation of the thoracic aorta by patch graft aortoplasty. J Thorac Cardiovasc Surg 1974; 68: 696-704.
11. Fleming WH, Sarafian LB, Clarck EB et al. Critical aortic coarctation: patch aortoplasty in infants less than age 3 months. Am J Cardiol 1979; 44: 687-90.

12. Hehlein FW, Mulch J, Rautenburg HW, Schlepper M, Scheld HM - Incidence and pathogenesis of late aneurysms after patch graft aortoplasty for coarctation. J Thorac Cardiovasc Surg 1986; 92: 22630.

13. DeSanto A, Bills RG, King $H$, Waller B, Brown JW - Pathogenesis of aneurysm formation opposite prosthetic patches used for coarctation repair: an experimental study. J Thorac Cardiovasc Surg 1987; 94:720-3.

14. Russell GA, Berry PJ, Watterson K, Dhasmana JP, Wisheart JD - Patterns of ductal tissue in coarctation of the aorta in the first three months of life. $J$ Thorac Cardiovasc Surg 1991; 102: 596-601.

15. Jones RA - Coarctation: do we need to resect ductal tissue? Ann Thorac Surg 1991; 52: 604-7.

16. Van Son JA, Falk V, Schneider P, Smedts F, Mohr FW - Repair of coarctation of the aorta in neonates and young infants. J Card Surg 1997; 12:13946.

17. Vouhé PR, Trinquet F, Lecompte $Y$ et al. - Aortic coarctation with hypoplastic aortic arch: results of extended end-to-end aortic arch anastomosis. J Thorac Cardiovasc Surg 1988; 96: 557-63.

18. Van Heurn LW, Wong CM, Spiegelhalter DJ et al. - Surgical treatment of aortic coarctation in infants younger than three months: 1985 to 1990 . Success of extended end-to-end arch aortoplasty. $J$ Thorac Cardiovasc Surg 1994; 107: 74-86.

19.Zehr KJ, Gillinov AM, Redmond JM et al. - Repair of coarctation of the aorta in neonates and infants: a thirty-year experience. Ann Thorac Surg 1995; 59: 3341.

20. Rajasinghe HA, Reddy VM, van Son JA et al. Coarctation repair using end-to-side anastomosis of descending aorta to proximal aortic arch. Ann Thorac Surg 1996; 61: 840-4.

21. Jacobsen JR, Wennevold A, Boesen I Coarctation of the aorta operated upon in infancy: longterm follow-up. Eur J Cardiol 1979; 10: 123-9. 
22. Campbell J, Delorenzi R, Brown J et al. Improved results in newborns undergoing coarctation repair. Ann Thorac Surg 1980; 30: 273-80.

23. Bergdahl LA, Blackstone EH, Kirklin JW, pacifico AD, Bargeron LM Jr. - Determinants of early success in repair of aortic coartaction in infants. $J$ Thorac Cardiovasc Surg 1982; 83: 736-42.

24. Brouwer MH, Kuntze CE, Ebels T, Talsma MD, Eijgelaar A - Repair of aortic coarctation in infants. $J$ Thorac Cardiovasc Surg 1991; 101:1093-8.

25. Todd PJ, Dangerfield PH, Hamilton DI, Wilkinson $\mathrm{JL}$ - Late effects on the left upper limb of subclavian flap aortoplasty. J Thorac Cardiovasc Surg 1983; 85: 678-81.

26. Geiss D, Williams WG, Lindsay WK, Rowe RD - Upper extremity gangrene: a complication of subclavian artery division. Ann Thorac Surg 1980; 30: 487-9.

27. Fenchel G, Steil E, Seybold-Epting W, Seboldt H, Apitz J, Hoffmeister HE - Repair of symptomatic aortic coarctation in the first three months of life: early and late results after resection and end-to-end anastomosis and subclavian flap angioplasty. $J$ Cardiovasc Surg 1988; 29: 257-63.
28. Han MT, Hall DG, Maché A, Rittenhouse EA Repair of neonatal aortic coarctation. J Pediatr Surg 1995; 30:709-12.

29. Conte S, Lacour-Gayet F, Serraf A et al. Surgical management of neonatal coarctation. $J$ Thorac Cardiovasc Surg 1995; 109: 663-75.

30. Uddin MJ, Haque AE, Salama AL, Uthman BC, Abushaban LA, Shuhaiber HJ - Surgical management of coartaction of the aorta in infants younger than five months: a study of fifty-one patients. Ann Thorac Cardiovasc Surg 2000; 6:252-7.

31. Cobanoglu A, Teply JF, Grunkemeier GL, Sunderland CO, Starr A - Coarctation of the aorta in patients younger than three months: a critique of the subclavian flap operation. $J$ Thorac Cardiovasc Surg 1985; 89:128-35.

32. Cobanoglu A, Thyagarajan GK, Dobbs JL Surgery for coarctation of the aorta in infants younger than 3 months: end-to-end repair versus subclavian flap angioplasty: is either operation better? Eur $J$ Cardiothorac Surg 1998; 14:19-26.

33. Wu JL, Leung MP, Karlberg J, Chiu C, Lee J, Mok CK - Surgical repair of coarctation of the aorta in neonates: factors affecting early mortality and recoarctation. Cardiovasc Surg 1995; 3:573-8. 